\title{
Dynamics of excitonic photoluminescence lineshape in narrow GaAs single quantum wells
}

\author{
K. FUJIWARA, R. CINGOLANI* and K. PLOOG** \\ Kyushu Institute of Technology, Tobata, Kitakyushu 804, Japan \\ * University of Lecce, Strada per Arnesano, 73100 Lecce, Italy \\ ** Paul-Drude-Institut für Festkörperelektronik, 1086 Berlin, Germany
}

\begin{abstract}
[Abstract] Dynamics of excitonic photoluminescence (PL) lineshape in narrow GaAs single quantum wells is investigated by measuring transient PL spectra in the picosecond time domains under direct excitation. We obtain distinct spectroscopic evidences for the exciton center-of-mass motion by drift-diffusion towards the local potential minima caused by the well-width fluctuations. That is, dynamical Stokes-shifts are directly observed. We find that inhomogeneous potential fluctuations in the quantum well plane result in asymmetry of the PL lineshape. The exciton trapped by the local minima formed due to the spatial inhomogeneity is directly evidenced by the lineshape analysis. From comparison with a case of spatially coherent quantum wells with growth island terraces, excellent correlation is obtained between the dynamical lineshape and the microscopic details of atomic-scale heterointerfaces.
\end{abstract}

\section{Introduction}

In narrow single quantum wells prepared by conventional MBE growth, a single broad photoluminescence (PL) peak is usually observed at low temperatures, which is located below the free heavyhole exciton resonance absorption line. Origin of this Stokes-shift is due to fast trapping of excitons by potential minima within the distributed exciton band, caused by the lateral well-width fluctuations[1-6]. Thus, radiative recombinations mostly occur via localized excitons, revealing the inhomogeneously broadened linewidth. In narrower wells[7], changes of the quantum mechanical confinement energy are larger by monolayer fluctuations, resulting in the larger linewidth and stokes-shift. Therefore, this provides a keen test of the heterointerface quality and pronounced effects of exciton trapping. In this paper, dynamics of excitonic PL lineshape is investigated in narrow GaAs/AlGaAs single quantum wells (SQW's) with and without interface disorder. By measuring transient PL spectra in the picosecond time domains under direct excitation, it is found that inhomogeneous lateral motions of excitons result in asymmetry of the PL lineshape. Excellent correlation is obtained between the atomicscale heterointerfaces and the exciton motions envisaged by the dynamical lineshape variations. 


\section{Experimental}

Two GaAs SQW samples were grown by molecular beam epitaxy (MBE) without and with growth interruption. In each sample, three different GaAs SQW's were contained with nominal $L_{z}$ values of $35 \AA, 55 \AA$ and 78 $\AA$. They were cladded by $360 \AA \mathrm{Al}_{\mathbf{x}} \mathrm{Ga}_{1-\mathrm{x}}$ As $(x=0.17)$ barriers and further confined by thick $(\sim 0.2 \mu \mathrm{m}) \quad \mathrm{Al}_{\mathrm{x}} \mathrm{Ga}_{1} \mathrm{x}$ As $(\mathrm{x}=0.3)$ layers to avoid surface recombinations. Time and spectral resolved PL experiments were performed by using a streak-camera based system and a pyrigine 2 dye laser synchronously pumped by a mode-locked $\mathrm{Ar}^{+}$laser[6]. The narrow GaAs SQW samples were mounted in a temperature variable cryostat and directly excited by 10 ps optical pulse with an energy $\sim 1.680$ eV $(738 \mathrm{~nm})$ which was transparent to the barriers. The low intensity cw PL and PL excitation (PLE) measurements were made at $6 \mathrm{~K}$ using the monochromatic radiation from growth interruption. a halogen lamp and a photon counting system.

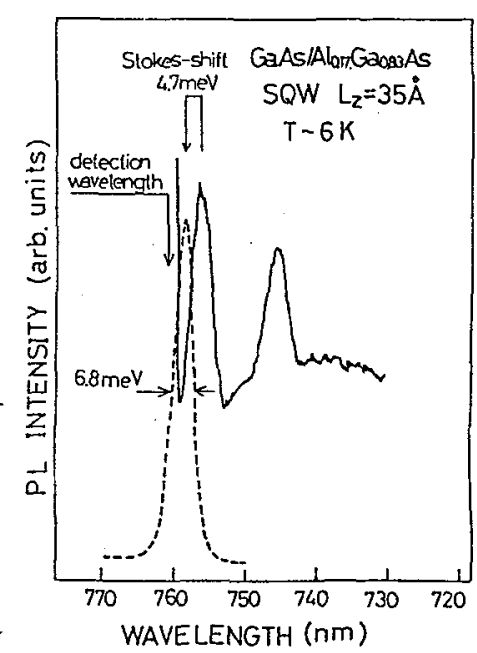

\section{Results and Discussion}

PL and PLE spectra are depicted in Fig. 1 of a $\mathrm{L}_{\mathrm{z}}=35 \AA$ GaAs SQW prepared without growth interruption. A single PL peak with a linewidth of $6.8 \mathrm{meV}$ (FWM) is observed, Stokes-shifted by $4.7 \mathrm{meV}$ (SS). A ratio of SS/FWL is 0.69 and is in accordance with the theory[8]. When the $L_{z}$ is increased, the $P L$ linewidth decreases, accompanying the reduced stokes-shift. These confirm the PL spectral characteristics originating from microscopic, statistical variations of the fluctuating well width as well as of the alloy composition in the barriers.

Dynamical changes of the PL spectra were measured for the GaAs SQW samples at $\sim 15 \mathrm{~K}$ and $\sim 80 \mathrm{~K}$. In order to allow fast momentum relaxation (fast exciton cooling) and to avoid photoexcited carriers transfer from the barriers (which complicates recombination time behaviors), optical pulse excitation was made at an energy of $\sim 1.680$ $\mathrm{eV}$, which is transparent to the barriers and more than 40 meV above the heavy-hole exciton resonance energy at $\sim 15 \mathrm{~K}$. Examples of transient PL spectra (in log and relative scale) are shown in Fig. 2 for the $\mathrm{L}_{\mathrm{z}}=35 \AA \mathrm{S} Q \mathrm{~W}$. Although their lineshape is broad, distinct peak shifts to longer wavelength sides, that is, dynamical stokesshifts are clearly observed. At the initial stages (up to $\sim 100$ ps), the PL lineshape is nearly symmetric, but at later times it becomes to be asymmetric as a result of reducing higher energy emissions preferentially (please note the changes of the PL spectra 3-5 in Fig. 2(b)). That is, emission-energy-dependent time behaviors[6] were observed. These results directly indicate the exciton centerof-mass motion by drift-diffusion towards the local potential 
minima, while they radiatively recombine. At $\sim 80 \mathrm{~K}$, on the other hand, such dynamical stokes-shifts and emission-energydependence were absent in the PL spectra in agreement with the previous ow studies $[4,9]$.

The time evolution is rigorously explained by a rate equation model which takes into account the exciton transfer within the inhomogeneously broadened exciton band. For this purpose, a single distributed exciton band is constructed using the PLE lineshape. Adopting the PI lineshape model proposed by schubert and Tsang for alloy semiconductors[10], we assume changes of the exciton concentrations at a given energy and time can be expressed by a nonlinear first-order partial differential equation. Details of our calculations will be described elsewhere. The calculated results are also shown in Fig. 2. Assuming an exciton trapping time constant of $\tau_{t}=140$ ps and a lifetime of radiative recombinations of $r,=500 \mathrm{ps}$, the stokes-shifts as well as the PL intensity changes are quantitatively well explained. We note however that there is an important difference in the lineshape asymmetry between theory and experiment at later times than $\tau$. (absence of high energy tails in the theory). This comes fron the assumed single homogeneously distributed exciton band which is not valid for the actual cases. The observed higher energy emissions mean that the excitons cannot escape once trapped by the local potential minima non-uniformly distributed. In this case, therefore, the PL decays are approximated by a single exponential time constant (radiative lifetime) only at later times, being consistent with our previous observation[6].

The time evolution of exciton recombinations and trapping events is more directly and elegantly envisaged by studying the time behaviors for the spatially coherent SQW prepared by MBE growth inter-. ruption. The time evolution of the PL spectra at $\sim 15 \cdot \mathrm{K}$ is shown in Fig. 3. Because, in this SQW, the lateral size of the terraces is 
much larger than the exciton Bohr radius, split exciton PL lines are observed corresponding to flat SQW islands (A, B, and C). Two stages of the lineshape asymmetry are seen in these transient PL spectra. That is, it is evidently shown that excitons transfer from the narrower wells ( $C$ and $B$ ) to the wider wells $(B$ and $A$ ), e.g., the inter-growth islands exciton transfer, as well as the intra-growth island transfer from the recombinations at. the terraces (free excitons, $F$ in Fig. 3) to the localized excitons, $L$ as illustrated in the lower time-integrated PL spectrum. These experimental results directly show the existence of micro-roughnesses on the macroscopically flat island terraces which supports the bimodal interface roughness model[11,12] for the atomic-scale structures of the GaAs/AlGaAs quantum well heterointefaces.

Fig. 3 Time resolved PL spectra measured at $\sim 15 \mathrm{~K}$ of the $\mathrm{L}_{\mathrm{z}}=35 \AA$ GaAs SQW sample prepared with 2 minutes growth interruption. Experimental spectra are displayed for 15 ps time integration and after the mean time: (1)45ps, (2)195ps, (3)570ps. Time-integrated spectrum is shown by a dashed one.

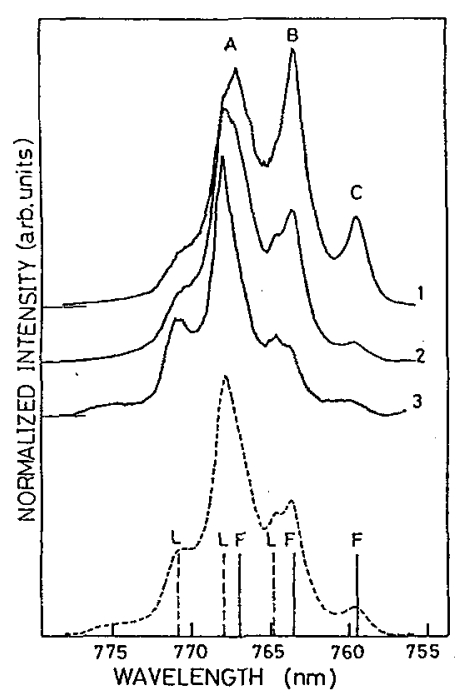

\section{Summary}

Dynamics of excitonic photoluminescence lineshape is studied in narrow GaAs/AlGaAs single quantum wells. The observed time behaviors as well as the lineshape asymmetry directly evidence the exciton lateral motions towards the local potential minima formed by the inhomogeneous well-width fluctuations. The existence of micro-roughnesses on the macroscopically flat quantum well island terraces are demonstrated by the lineshape analysis.

References

[1] Weisbuch C., Dingle R., Gossard A.C. and Wiegmann W., solid state Commun. 38 (1981) 709-712.

[2] Bastard G., Delalande C., Meynadier M.H., Frijlink P.M. and Voos M., Phys. Rev. B29 (1984) 7042-7044.

[3] Hegarty J., Goldner L. and Sturge M.D., Phys. Rev. B30 (1984) 7346-7348.

[4] Delalande C., Meynadier M.H. and Voos M., Phys. Rev. $B 31$ (1985) 2497-2498.

[5] Fujiwara K., Kanamoto K., Tsukada N., Miyatake H. and Koyama H., J. Appl. Phys. 66 (1989) 1488-1491.

[6] Fujiwara K., Katahama H., Kanamoto K., Cingolani R. and Ploog K., Phys. Rev. B43 (1991) 13978-13982.

[7] Zachau M., Kash J.A. and Masselink W.T., Phys. Rev. B44 (1991) 8403-8406.

[8] Yang F., wilkinson M., Austin E.J. and O'Donnell K.P., Phys. Rev. Lett. 70 (1993) 323-326.

[9] Fujiwara K., Kanamoto K. and Tsukada N., Phys. Rev. B40 (1989) 9698-9702.

[10] Schubert E.F. and Tsang W.T., Phys. Rev. B34 (1986) 2991-2994.

[11] Gammon D., Shanabrook B.V. and Katzer D.S., Phys. Rev. Lett. 67 (1991) 1547-1550.

[12] Warwick C.A. and Kopf R.F., Appl. Phys. Lett. 60 (1992) 386-388. 